\title{
Diagnostik und störungsspezifische Therapie bei Sexualdelinquenten mit psychischen Störungen
}

\section{Diagnosis and Disorder-Specific Therapy in Sex Offenders with Mental Disorders}

\author{
$\begin{array}{lll}\text { J. Hoyera } & \text { B. Borchard } \\ \text { b H. Kunst } & \text { H }\end{array}$
}

aKlinische Psychologie und Psychotherapie, TU Dresden, bNiedersächsisches Landeskrankenhaus Moringen, 'Institut für Psychologie, Johann Wolfgang Goethe Universität, Frankfurt am Main

\section{Schlüsselwörter \\ Sexualdelinquenten · Paraphilie · Störung der Impulskontrolle $\cdot$ Störungsspezifische Therapie}

\section{Zusammenfassung}

Hintergrund: Systematische Darstellungen zur Diagnostik und Verhaltenstherapie von sexuellen Gewalttätern mit psychischen Störungen fehlen im deutschen Sprachraum fast völlig. In der vorliegenden Arbeit werden deshalb für diese kleine, aber besonders gefährliche Tätergruppe die auf der Basis des DSM IV hauptsächlich relevanten Diagnosen (Paraphilie und Störung der Impulskontrolle) und für diese Störungen geeignete Behandlungsmethoden vorgestellt.

Methoden: Merkmale, die Paraphile und Impulskontrollgestörte im Hinblick auf Lerngeschichte, sexuelle Präferenz und tatauslösende Bedingungen differenzieren, werden dabei hervorgehoben. Weiterhin wird eine erste Validierungsstudie vorgestellt, in der untersucht wurde, inwieweit sich beide Störungsgruppen hinsichtlich Impulsivität, sozialer Angst, Konfliktvermeidung und dysfunktionaler Selbstaufmerksamkeit unterscheiden.

Ergebnisse: Die Ergebnisse verdeutlichen, dass die vorgeschlagene Kategorisierung valide ist und dass sich Paraphile und Impulskontrollgestörte psychologisch bedeutsam voneinander differenzieren lassen.

Schlussfolgerungen: Auf dieser Basis lassen sich Aussagen über störungsspezifisch relevante Behandlungselemente ableiten. Unterschiedliche Vorgehensweisen bei Paraphilen bzw. Impulskontrollgestörten werden für folgende Bereiche skizziert: Aufbau einer therapeutischen Beziehung, Änderung der sexuellen Präferenzen, Training sozialer Fertigkeiten und der Konfliktbewältigung, Modifikation dysfunktionaler Kognitionen und Rückfallprävention.

\author{
Key Words \\ Sexual offenders · Paraphilia - Impulse control disorder . \\ Disorder specific therapy
}

\section{Summary}

Background: Systematic work regarding the diagnostics and behavior therapy of violent sex offenders with mental disorders is mostly lacking in German publications. Therefore, this study presents the diagnoses which are mainly relevant for this small but particularly dangerous group of offenders on the basis of DSM IV (paraphilia and impulse control disorders) and describes specifically indicated interventions.

Methods: Characteristic features are emphasized which differentiate paraphiliacs and offenders with an impulse control disorder with regard to learning history, sexual preferences, and the stimulus conditions which triggered the offense. Further, a validation study is presented which examined the differences between both disorders concerning impulsivity, social anxiety, avoidance of interpersonal conflicts, and dysfunctional self-consciousness.

Results: Results showed that the proposed categorization is valid and that paraphiliacs and offenders with an impulse control disorder can be psychologically useful differentiated.

Conclusions: In this framework, first conclusions can be drawn regarding the treatment elements which are considered to be relevant for disorder-specific therapy. Different procedures for the treatment of paraphiliacs and offenders with an impulse control disorder are described for the following areas: establishment of a therapeutic relationship, modification of sexual preferences, training of social and coping skills, conflict management, modification of cognitive distortions, and relapse prevention.

\section{KARGER \\ Fax +497614520714 \\ (c) 2000 S. Karger GmbH, Freiburg \\ E-mail Information@Karger.de \\ www.karger.com}

Dipl. Psych. Dr. phil. habil. Jürgen Hoyer

Technische Universität Dresden

Klinische Psychologie und Psychotherapie

D-01062 Dresden (Deutschland) 


\section{Einleitung}

Der größte Teil der Sexualstraftäter weist keine delikt- oder vollzugsrelevanten psychischen Störungen auf, er ist als kriminell zu klassifizieren. Die Gruppe der Sexualstraftäter, die wiederholt schwerwiegende sexuelle Delikte einschließlich körperlicher Gewalt ausüben und aufgrund mitbedingender psychischer Störungen in der forensischen Psychiatrie unterzubringen sind, ist demgegenüber zahlenmäßig klein [vgl. Schorsch und Pfäfflin, 1994]. Durch die hohe Rückfallneigung der letztgenannten Täter ist jedoch der Anteil der von ihnen begangenen Taten (Vergewaltigung, Kindesmissbrauch, sexuelle Tötungsdelikte) überproportional hoch. Ihre Taten erfahren außerdem ein erhebliches Maß an Aufmerksamkeit in der Öffentlichkeit und, was schwerer wiegt, führen oft zu besonders starker Schädigung der Opfer und ihrer Angehörigen [vgl. Amann und Wipplinger, 1997].

Aufgrund der beträchtlichen Relevanz, die einer effektiven Behandlung dieser Täter zukommt, wären erhebliche Forschungsaktivitäten zu (1) Fragen der klinisch-psychologischen Deskription und Erklärung ihrer Störungen, (2) zur psychologisch-psychiatrischen Diagnostik und (3) zur Erprobung und Evaluation von (spezifischen) Behandlungsmaßnahmen erforderlich. Tatsächlich ist in geradezu eklatanter Weise das Gegenteil der Fall:

- Es gibt nur wenige Untersuchungen zur Frage der Prävalenz von Achse-I-Störungen oder Persönlichkeitsstörungen bei dieser spezifischen Tätergruppe [z.B.: Berner et al., 1992], und diese beziehen sich auf unbefriedigend kleine Stichproben.

- Weil es zu wenige diagnostisch befriedigende Studien gibt, kann es auch keine störungsspezifischen Erklärungsmodelle geben - oder es werden psychologische Erklärungsmodelle entwickelt [z.B. Ward et al., 1995], auf empirische Prüfung wird aber verzichtet.

- Und schließlich fehlen Studien zur Evaluation von Behandlungsmaßnahmen, insbesondere Katamnesen mit einem sehr langen Erfassungszeitraum. [Für eine ähnliche Bewertung des Forschungsstandes vgl. Hoyndorf et al., 1995.]

Diese Kritik bezieht sich jedoch nur auf die zuvor eingegrenzte Tätergruppe. $\mathrm{Zu}$ anderen im Zusammenhang mit sexuell-delinquenten Handlungen relevanten Störungen, wie Exhibitionismus oder weniger fremdgefährdenden Formen der Pädophilie, liegen durchaus befriedigende Forschungsergebnisse vor, die eine differentiell verhaltenstherapeutische Behandlung begründen können. Hier handelt es sich jedoch um sogenannte Hands-off-Delikte; diese Täter können häufig ambulant behandelt werden.

Für das Problem schwerer Sexualdelikte sind die Resultate vorliegender Forschung - auch international - aber auch aus einem anderen Grund nur eingeschränkt brauchbar: Die meisten Untersuchungen weisen ein kardinales methodisches Problem auf. Sie gehen in der Regel nach juristischen oder
Einsichts- und Steuerungsfähigkeit

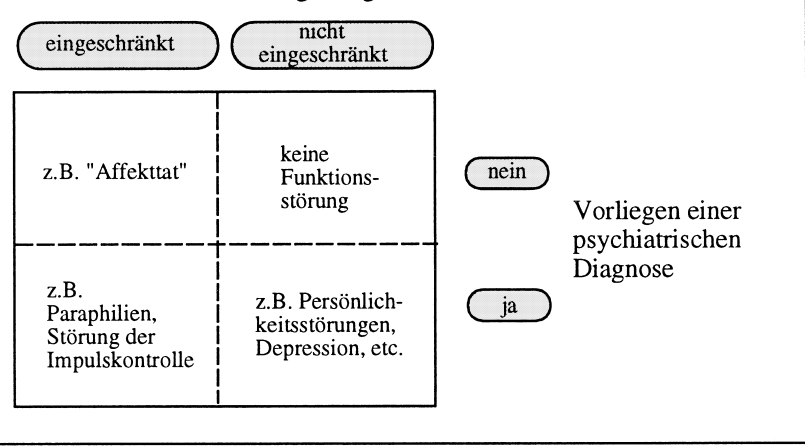

Abb.1. Sexualstraftäter: Klassifikation nach Einsichts- und Steuerungsfähigkeit und Vorliegen psychiatrischer Diagnosen.

kriminologischen Kategorien vor, und Art und Ausmaß eventuell vorliegender psychopathologischer Störungen bleiben unklar [vgl. Hoyer et al., 1999; Okami und Goldberg, 1992]. Ziel des vorliegenden Beitrags ist es deshalb, aufbauend auf einer eigenen Studie einen Klärungsvorschlag zur Diagnostik psychischer Störungen in dieser Tätergruppe zu machen, aus dem sich Indikationen für eine störungsspezifische Behandlung ableiten lassen. Dabei werden vor allem zwei diagnostische Kategorien herangezogen - Paraphilie und Störung der Impulskontrolle. Störungsdeskriptive Merkmale, differentielle Lerngeschichte, sexuelle Präferenz und störungsspezifische Behandlung werden beschrieben.

\section{Sexualdelinquenz: Kriminologische versus psychologische Perspektive}

Die für den vorliegenden Beitrag relevante Gruppe besteht aus Sexualdelinquenten, die aufgrund einer psychischen Störung zum Tatzeitpunkt eine eingeschränkte Einsichtsoder Steuerungsfähigkeit aufweisen und deswegen gemäß $\$ 63$ StGB im Maßregelvollzug bzw. in der forensischen Psychiatrie «Maßnahmen zur Besserung und Sicherung» zuzuführen sind. Abbildung 1 verdeutlicht, dass sich die Merkmale «psychische Störung» und «mangelnde Einsichts- oder Steuerungsfähigkeit» bei dieser Gruppe überlappen, aber auch einzeln auftreten können.

Die internationale Forschung zur Sexualdelinquenz zieht in der Regel weder das Kriterium der psychischen Störung noch das der eingeschränkten Einsichts- oder Schuldfähigkeit heran. Vielmehr ist die Differenzierung in Vergewaltiger (rapists) und in Missbrauchstäter (child molesters) gebräuchlich [vgl. Marshall et al., 1990; auch: Rehder, 1996a,b]. Aus unserer Sicht handelt es sich hierbei um eine kriminologische Forschungsperspektive, die relevant ist, wenn man Aussagen über die Gesamtheit der Täter machen will, also eine Gruppe, 
die nur zum Teil behandlungsbedürftig ist. Beschränkt man sich auf die Untersuchung der Täter mit psychischen Störungen, so ist ein Perspektivenwechsel jedoch unumgänglich, da die Gruppen der Vergewaltiger und der Missbrauchstäter psychodiagnostisch inhomogen sein können (s. unten). So weisen zum Beispiel nicht alle Vergewaltiger oder Missbrauchstäter ein und dasselbe abweichende Muster sexueller Präferenz und Erregung auf.

Nur für Paraphile gilt, dass auffälliges Sexualverhalten ausgeübt wird, weil es zur stärksten Erregung und damit zur stärksten Befriedigung führt, und dass die Erregung von spezifischen (devianten) Stimuli oder Verhaltensweisen abhängig ist. Entsprechende Handlungsimpulse treten über längere Zeiträume hinweg perseverierend, vor allem in Belastungssituationen, auf. Die Betroffenen spüren diese Impulse, kämpfen dagegen an, sind aber nicht in der Lage, sie zu kontrollieren. Relevante Diagnosen aus der Kategorie der Paraphilien sind Fetischismus, Exhibitionismus, Pädophilie und Sadismus (s. Tab. 1 für die diagnostischen Kriterien der Paraphilie), die zu unterschiedlichen Delikten führen können, aber nicht müssen.

Untersuchungen zeigen jedoch, dass nur ein Teil der gefährlichen Sexualstraftäter derartige Störungen aufweist. Nur etwa ein Drittel der Männer, die wegen Kindesmissbrauchs auffällig wurden, zeigt eine pädophile Präferenz [Barbaree und Marshall, 1989]. Die Mehrzahl der Vergewaltiger wird auch durch Stimuli einer einwilligenden Partnerin erregt [vgl. Baxter et al., 1986; Barbaree, 1990]. Selbst wenn man berücksichtigt, dass die Validität solcher Studien aufgrund von Verleugnungstendenzen der Probanden eingeschränkt sein könnte, so belegen sie doch deutlich, dass Sexualstraftäter eine eher heterogene Gruppe darstellen, und damit gilt: «Sexualdelikte per se gestatten keine Diagnose» [Hoyndorf et al., 1995, S.111].

Die mit dem bisher Gesagten belegte Heterogenität von Gruppen von Sexualdelinquenten wird in der Forschung allerdings allzu selten berücksichtigt. Mit einer Forschungsstrategie, die eher an juristisch oder kriminologisch relevanten Deliktkategorien orientiert ist, wird das Problem der psychologischen oder psychodiagnostischen Heterogenität der Gruppen nicht gelöst, selbst wenn die Strategie sehr ausgefeilt ist. Vergewaltiger wie Missbrauchstäter können paraphil sein oder nicht: Unterschiedliche Ergebnisse zwischen Studien ergeben sich allein aufgrund des zufälligen und nicht dokumentierten Anteils Paraphiler (oder anderer diagnostischer Subgruppen) an den vorgefundenen Stichproben.

Eine Forschungspraxis, die trotzdem so tut, als ob Gruppen von Vergewaltigern oder Missbrauchstätern diagnostisch homogen seien, muss zwangsläufig zu uneinheitlichen Ergebnissen führen [vgl. Okami und Goldberg, 1992] und in der Folge zur Resignation, was die empirisch begründete Möglichkeit zu einer störungsspezifischen Behandlung anbelangt.

Für dieses Problem möchten wir im vorliegenden Beitrag einen Lösungsvorschlag machen, aus dem sich unseres Erach-
Tab. 1. Diagnostische Kriterien der Paraphilie und Störung der Impulskontrolle nach DSM IV

Diagnostische Kriterien der Paraphilie

Wiederkehrende, intensive sexuell erregende Phantasien, sexuell drang-

hafte Bedürfnisse oder Verhaltensweisen, die sich im allgemeinen auf:

1) nichtmenschliche Objekte

2) das Leiden oder die Demütigung der eigenen Person oder des Partners

3) Kinder bzw. andere Personen richten, die mit der sexuellen Interaktion nicht einverstanden sind oder wären.

Diagnostische Kriterien der Störung der Impulskontrolle n.n.b.

1) die Unfähigkeit einem Impuls zu widerstehen, der für die Person oder andere schädlich ist

2) ein zunehmendes Gefühl von Spannung oder Erregung vor der Durchführung der Handlung

3) das Empfinden von Befriedigung oder Erleichterung während der Durchführung

tens Ansatzpunkte für sicherere Indikationsentscheidungen in der therapeutischen Behandlung ergeben.

Unsere Ausgangsüberlegung: Wenn nicht alle Täter eine Paraphilie aufweisen, welche andere Störung dann? Die Antwort kann nicht im Verweis auf das Vorliegen einer Persönlichkeitsstörung bestehen, denn diese allein reicht nicht aus, um zu erklären, warum jemand wiederholt gefährliche Sexualstraftaten begeht (womit wir uns im übrigen in Übereinstimmung mit dem Strafrecht befinden; vgl. demgegenüber kritisch: Kröber, 1997). Wichtiger für die Erklärung solcher Taten finden wir vielmehr den Umstand, dass die Täter - und eben nicht nur die Paraphilen - in der Tatsituation keine normale Erregungshemmung aufweisen. Aggressive oder sexuelle Impulse können nicht mehr kontrolliert werden [vgl. Disinhibitions-Theorie; Barbaree et al., 1979; Quinsey et al., 1984]. Dieser diagnostische Sachverhalt - mangelnde Erregungshemmung - findet sich im DSM IV nicht nur bei der Kategorie Paraphilie, sondern auch bei der Kategorie Störung der Impulskontrolle (vgl. Tab. 1 für die diagnostischen Kriterien). Relevant ist im vorliegenden Kontext die Restkategorie der nicht näher bezeichneten Impulskontrollstörung (DSM IV 312,30), da im DSM IV keine eigene Kategorie für Impulskontrollstörungen mit sexueller Aggression vorgesehen ist.

Bereits Berner und Karlick-Bolten [1985, ähnlich: Schorsch und Becker, 1977] haben darauf hingewiesen, dass es die (heutigen DSM-IV-)Kategorien der Paraphilie auf der einen und die nicht näher bezeichnete Störung der Impulskontrolle auf der anderen Seite erlauben, eine diagnostisch valide Grobdifferenzierung von Tätern mit schweren Sexualstraftaten vorzunehmen, die auch für die Entwicklung differentieller Störungs- und Behandlungsmodelle heuristisch relevant ist. Demgegenüber beschreibt eine Persönlichkeitsstörung ein «überdauerndes Muster von innerem Erleben und Verhalten» 
Tab. 2. Delikte und Diagnosen bei den untersuchten Sexualstraftätern $(\mathrm{N}=68)$

\begin{tabular}{llc}
\hline & Vergewaltigung & Kindesmissbrauch \\
\hline Impulskontrollgestörte & 21 & 5 \\
Paraphile & 14 & 28 \\
\hline
\end{tabular}

einer Person [DSM IV; Saß et al., 1996, S.711], ohne exakte Vorhersagen darüber zu formulieren, ob ein Verhalten in bestimmten Situationen auftritt oder nicht. Die Neigung zu impulsiven Handlungen gilt als unspezifisches Symptom vieler Persönlichkeitsstörungen, das aber nicht auftreten muss. Die Diagnose einer Impulskontrollstörung erlaubt demgegenüber eine differenziertere Beschreibung, Beurteilung und Prognose des Problemverhaltens.

Komorbide Persönlichkeitsstörungen (insbesondere antisoziale, narzisstische und Borderline-Persönlichkeitsstörungen) müssen selbstverständlich ebenfalls erfasst werden, da sie erhebliche Auswirkungen auf Therapieerfolg, Therapiekrisen [vgl. Fiedler, 1994] und auf die Prognose haben; sie sind aber keine geeigneten Kategorien für die Beurteilung der Einsichts- und Steuerungsfähigkeit zum Tatzeitpunkt. Außerdem liegt ein besonderes Problem bei der Diagnostik von Persönlichkeitsstörungen immer noch in der Reliabilität von differentialdiagnostischen Entscheidungen. Über die Tatsache, dass überhaupt eine Persönlichkeitsstörung vorliegt, lässt sich mit größerer Sicherheit Einvernehmen erzielen, als über die Frage, welche vorliegt [vgl. Fydrich et al., 1996].

Weitere Diagnosen psychisch gestörter aggressiver Sexualstraftäter sind die der Psychosen, der intellektuellen Minderbegabung oder Hirnschädigung [vgl. Schüler-Springorum et al., 1996]. Auf das Problem der spezifischen Behandlung dieser Patienten wird jedoch im vorliegenden Beitrag nicht eingegangen.

\section{Paraphilie und Impulskontrollstörung: Neue Ergebnisse}

In einer eigenen Studie haben wir an 72 im Maßregelvollzug untergebrachten Sexualstraftätern untersucht, ob eine Einteilung von Sexualstraftätern nach den beiden vorgeschlagenen psychodiagnostischen Kategorien psychometrisch valide ist und hinsichtlich relevanter psychologischer Variablen mehr Varianz aufklärt als eine Aufteilung nach Delikten.

Zur Gruppenbildung haben wir dabei die Patientenakten herangezogen. In den dort dokumentierten psychiatrischen Gutachten wurde der Tathergang wie auch das Vorliegen spezifischer sexueller Phantasien jeweils genau dokumentiert, was uns erlaubte zu beurteilen, ob:

(1) paraphile Phantasien vorlagen und ausgeführt wurden (Kriterium für die Diagnose Paraphilie) oder ob

(2) diese nicht vorlagen und für den Tathergang ein Verlust der Impulskontrolle gegeben war (Kriterium für Impulskontrollstörung nnb).

Mit dieser (dichotomen) Kategorisierung konnten wir fast alle $(94 \%)$ der beurteilten Täter einer Gruppe zuordnen. Der erste wichtige Befund dabei war, dass die Frage, ob eine Paraphilie vorliegt oder nicht, sich tatsächlich als unabhängig vom Delikt erwiesen hat (Tab. 2). Die Daten zeigen auch, dass Sadisten in der Gruppe der Vergewaltiger keineswegs selten sind. Wenn in der Literatur vermutet wird, Sadisten unter den Vergewaltigern seien selten [Abel und Osborn, 1992; Brown, 1983], so gilt das vielleicht im Hinblick auf die Gesamtpopulation (von Vergewaltigern), offensichtlich aber nicht auf die im Maßregelvollzug.

Die Befunde (Tab. 2) bedeuten unseres Erachtens unmittelbar: Differentielle Behandlung kann sich nicht an Deliktgruppen, sondern muss sich an Diagnosegruppen orientieren. Wir haben diese Überlegung jedoch in der Studie auch einem empirischen Test unterzogen. Bringt eine Aufteilung nach Diagnosen eine bessere Varianzaufklärung als diejenige nach Delikten, wenn man die Ausprägung psychologisch relevanter Variablen betrachtet?

Als psychologisch relevant haben wir dabei auf der Basis der vorliegenden Literatur [vgl. dazu Hoyer et al., 1999] die folgenden Variablen erachtet:

- Impulsivität [Barratt, 1995],

- soziale Angst [Stangier und Heidenreich, 1997],

- Vermeidung interpersoneller Konflikte [Horowitz et al., 1994] und

- dysfunktionale Selbstaufmerksamkeit [Hoyer, 1996].

Diese Variablen wurden jeweils mittels Fragebogen erfasst, wobei wir ein Einzel-Untersuchungssetting gewählt haben. Entgegen dem Vorurteil, dass man mit Maßregelvollzugspatienten keine reliablen Fragebogendaten erhält, waren die Werte für die interne Konsistenz weitgehend befriedigend bis gut.

Die Ergebnisse der Diskriminanzanalyse zeigen, dass bei einer Aufteilung nach Delikten tatsächlich keinerlei Unterschiede hinsichtlich der Fragebögen zu entdecken sind, sich bei einer Einteilung nach Diagnosen jedoch signifikante Unterschiede ergeben. Bei einer Aufteilung nach Diagnosen werden bei signifikanter Diskriminanzfunktion $(\mathrm{p}=0,04)$ $72 \%$ der Probanden korrekt zugeordnet, bei einer Aufteilung nach Delikten liegt die Zuordnung bei insignifikanter Diskriminanzfunktion nicht über dem Zufallswert. Bemerkenswert an diesem Ergebnis scheint uns, dass die Zuordnung allein aufgrund psychometrischer Variablen erfolgte, die nicht in direktem Zusammenhang zu diagnostischen Merkmalen der Störung stehen. Die Relevanz dieser Variablen wurde vielmehr theoretisch aus ätiologischen Modellen abgeleitet, was den Einsatz dieser Modelle auch in der Praxis begründet [Hoyer et al., 1999]. Im Einzelnen waren besonders die soziale Angst und die Konfliktvermeidung zur Diskrimination geeignet; beide Variablen sind bei Paraphilen stärker ausgeprägt. 
Tab. 3. Präferenztyp, auslösende Bedingungen, Verstärkungsmodus: idealtypische Unterschiede bei paraphilen versus impulskontrollgestörten Sexualdelinquenten [vgl. Wulfert et al., 1996]

\begin{tabular}{llc}
\hline & Paraphile & Impulskontrollgestörte \\
\hline Sexuelle Präferenz & deviante Phantasie u. Handlung bevorzugt & Präferenz für «normale» Sexualität \\
Auslösende Bedingung & sexuelle Deprivation (hinsichtlich der devianten Stimuli) & $\begin{array}{l}\text { «deviantes Arousal» möglich, } \\
\text { situative Bedingungen mit Verstärkerverlust }\end{array}$ \\
Verstärkungsmodus & positive Verstärkung & negative Verstärkung (Spannungsreduktion) \\
\hline
\end{tabular}

Behandlungsrelevante, störungsdeskriptive Unterschiede

Welches sind die behandlungsrelevanten Unterschiede zwischen Paraphilen und Impulskontrollgestörten?

Wulfert et al. [1996] haben darauf hingewiesen, dass ein wesentlicher Unterschied zwischen sexuell eindeutig paraphil orientierten Tätern und solchen, die eher situationsabhängig handeln - diese Unterscheidung ähnelt der von uns getroffenen - in der jeweiligen Lerngeschichte und im unterschiedlichen Verstärkungsmodus der devianten sexuellen Handlungen liegt. Bei Paraphilen im engeren Sinne ist danach die deviante Handlung die für die Täter normale (bzw. die bevorzugte) sexuelle Aktivität. Auslösende Bedingungen für diese sexuellen Handlungen sind damit in sexueller Deprivation (und gegebenenfalls in entsprechenden Gelegenheiten) zu sehen. Sexuell deviantes Verhalten steht bei dieser Tätergruppe unter Bedingungen positiv operanter Verstärkung. Zusätzlich kann das sexuell deviante Verhalten auch der Reduktion von Versagensängsten dienen [vgl. Laws und Marshall, 1990].

Im Gegensatz dazu bevorzugen die nach unserer Diktion «impuls-kontrollgestörten Täter» per definitionem - sonst wären sie Paraphile - eine non-deviante sexuelle Betätigung. Auslösende Bedingungen dafür, dass sie sich auf sexuell deviante Weise betätigen, liegen nach Wulfert et al. [1996] in einem subjektiv schwerwiegenden Verstärkerverlust, der sich zum Beispiel auf eine sexuelle Beziehung, auf Arbeitsplatzverlust oder persönliche Kränkungen beziehen kann. Damit gehe oft ein (sekundärer) Verlust sozialer Verstärker und des Selbstwertgefühls einher. Für die Bewältigung der in den genannten Situationen auftretenden Stressspannung besitzen die Betroffenen keine oder unreife und inadäquate Bewältigungsmöglichkeiten. Die sexuell deviante Handlung dient bei diesen Tätern dem Spannungsabbau; sie steht unter den Bedingungen negativer Verstärkung (Tab. 3).

Diese Unterschiede hinsichtlich Lerngeschichte, sexueller Präferenz und Kontingenzverhältnis bieten eindeutige Anhaltspunkte für eine differentielle Behandlung. Die Behandlung (und die Prognose) für Täter mit sexuell devianter Präferenz, also für Paraphile, muss anders ausfallen als für Täter mit einer Impulskontrollstörung, deren sexuelle Orientierung nicht grundsätzlich gestört sein muss, sondern deren Problem eher darin liegen kann, dass sie intrapsychische Spannungssituationen oder persönliche Krisen nicht anders als durch Gewaltausübung bewältigen können [vgl. auch Marshall und Barbaree, 1990; Füllgrabe, 1994].

\section{Behandlungselemente unter störungsspezischer Perspektive}

Unter störungsspezischer Therapie verstehen wir mit Fiedler [1997, S.10] «die ätiologietheoretische Begründung des individuellen therapeutischen Vorgehens jeder anstehenden Psychotherapie (...). Dieses Vorgehen ist in der Herleitung therapeutischer Strategien und Methoden und im deutlichen Unterschied zur herkömmlichen Problemanalyse vorrangig deduktiv strukturiert, weil theoriegeleitet. Das Grundlagenwissen über Ätiologie, Verlauf und Veränderbarkeit psychischer Störungen (...) begründet therapeutische Strategien. Erst in der konkreten Abstimmung mit dem Patienten werden weitere, induktiv zu generierende methodische Konkretisierungen nötig.» Der Anspruch, in diesem Sinne störungsspezische Behandlung bei Sexualdelinquenten ätiologietheoretisch begründet abzuleiten, kann - wie wir bereits kritisiert hatten auf der Basis der bisher vorliegenden Forschungsevidenz keineswegs schon eingelöst werden. Wenn andererseits allein auf die Notwendigkeit einer individualisierenden Behandlung verwiesen wird [vgl. etwa Schüler-Springorum et al., 1996], wirkt das unseres Erachtens aber beliebig und hemmt Bemühungen, in der Behandlung von Sexualstraftätern spezifische Strategien zu beschreiben und zu evaluieren.

Wir möchten deshalb im Folgenden zumindest einige Vorschläge hierzu machen, die gewissermaßen als Heuristiken für Therapeuten $\mathrm{zu}$ verstehen sind. Weiterhin möchten wir verschiedene, in der Literatur als Standardmethoden in der Behandlung von Sexualdelinquenten genannte Therapieelemente vorläufig und hypothetisch im Hinblick darauf bewerten, inwieweit sie für die Gruppen der Paraphilen und der Impulskontrollgestörten indiziert sein dürften. Wir orientieren uns dabei weitgehend an der im Handbook of Sexual Assault [Marshall et al., 1990] gewählten Einteilung. 


\section{Förderung der Therapiemotivation und Aufbau einer tragfähigen therapeutischen Beziehung}

Leidensdruck und Veränderungsmotivation können bei beiden Störungsgruppen bestehen oder auch nicht; der Aufbau einer vertrauenvollen Therapiebeziehung ist aber unter den Bedingungen des Maßregelvollzugs prinzipiell erschwert [vgl. Jacobs et al., 1990] und sollte grundsätzlich unter der Supervision erfahrener Therapeuten erfolgen. Ergebnisse unserer eigenen Untersuchung erbringen im übrigen Hinweise, dass die Therapiemotivation bei Paraphilen eher höher ist. Ähnlich konnten Abel et al. [1988] zeigen, dass Täter mit multiplen «Diagnosen» (hinsichtlich hands-on/hands-off-Kategorisierung, Geschlecht des Opfers, Inzest/Nicht-Inzest), also Täter ohne deviante Fixierung, häufiger die Therapie abbrechen und auch häufiger rückfällig werden.

Störungsspezifisches Behandlerwissen kann in beiden Gruppen zum Aufbau einer therapeutischen Beziehung und zur Entwicklung von Therapiemotivation günstig sein. Es erleichtert zum einen dem Behandler das klinisch-psychologische Störungsverständnis und liefert damit eine Voraussetzung, sich nicht nur z.B. auf die aversiven Aspekte der Tat zu fixieren. Zum anderen kann ein ähnlicher Effekt auftreten wie bei einem Panikpatienten, der sich nach der Vermittlung eines rationalen Erklärungsmodells für sein Symptom zum ersten Mal «gut aufgehoben» fühlt und sich endlich in Händen eines professionellen Behandlers weiß. Befürchtungen, der Behandler könne mit dem Delikt auf nachteilige Weise umgehen, können so ausgeräumt werden. Es wird vertrauensbildend sein, wenn ein impulskontrollgestörter Täter merkt, dass sein Therapeut ihn eben nicht wie einen «Perversen» behandelt - und umgekehrt, wenn ein Paraphiler merkt, dass der Therapeut die Unabweisbarkeit und Hartnäckigkeit devianter Phantasien als handhabbare Störung auffasst und vor dem schwierigen Thema nicht auf andere Störungsaspekte ausweicht, die sich vielleicht im Stationsalltag zeigen.

\section{2. Änderung der sexuellen Präferenzen}

Impulskontrollgestörte unserer Definition weisen ein normales sexuelles Arousal auf und zeigen lediglich mangelnde Inhibition aggressiver oder sexueller Impulse. Es wäre im Gegensatz zu den Paraphilen nicht indiziert zu versuchen, im engeren Sinne ihre sexuellen Präferenzen zu verändern.

Überblicksartikel zu diesem Thema kommen allerdings noch nicht zu einem sicheren Urteil, ob und inwieweit sich die sexuellen Präferenzen überhaupt ändern lassen [vgl. Laws und Marshall, 1991; Seligman, 1993] bzw. welche Rekonditionierungsstrategien hier am vielversprechendsten sind. Diskutiert werden unter anderem verdeckte Sensibilisierung, masturbatorische Rekonditionierung und Thematic Shift, Aversionsund Satiations-Verfahren [Abel et al., 1992]. Während Erfolge dieser Methoden in Einzelfallberichten (bei Exhibitionis- ten und bei Tätern mit sexuellen Missbrauchsdelikten an Kindern) belegt sind, fehlen Studien an psychisch gestörten Gewalttätern noch weitgehend. Wichtig scheint hier die Beobachtung von Kockott [1996], dass sexuelle Deviationen meist mit erheblichen heterosexuellen Verhaltensdefiziten einhergehen und dass somit ein Abbau devianter Verhaltensmuster ohne einen parallelen Aufbau non-devianter, sexuell kompetenter Verhaltensweisen wenig erfolgversprechend sein dürfte [vgl. auch Hoyndorf und Christmann, 1994].

\section{Medikamentöse Behandlung}

Ähnlich wie bei anderen schweren psychischen Störungen kann eine medikamentöse Therapie unterstützend hilfreich sein [vgl. Horn, 1990; Raine, 1993]. Unsere diagnostische Differenzierung überschneidet sich zum Teil mit der psychiatrischen Unterscheidung von «echten» und «unechten» Triebtätern [Schuhmacher, 1990], und man kann sich Erfolg von triebdämpfenden Medikamenten nur bei einer «Triebproblematik» erhoffen. Ob Medikamente aber wirklich den so genannten Trieb dämpfen können, inwieweit die deviante Phantasietätigkeit vermindert werden kann, und inwieweit Effektivität im Hinblick auf Compliance und Rückfallgefahr angenommen werden kann, wird an anderer Stelle diskutiert [vgl. Abel et al., 1992]. Weiterhin gibt es Hinweise, daß bei impulskontrollgestörten Täter neurologische und/oder biochemische Auffälligkeiten vorliegen, die medikamentöser Behandlung offen stehen [Raine, 1993].

\section{Training sozialer Fertigkeiten und Konfliktbewältigung}

Die meisten Sexualstraftäter dürften im Bereich sozialer Fertigkeiten gravierende Defizite aufweisen. Wir können an dieser Stelle auf die oben erwähnte eigene Studie zurückkommen: Sie zeigte signifikante Unterschiede zwischen Paraphilen und Impulskontrollgestörten hinsichtlich der sozialen Angst und der Konfliktvermeidung in interpersonellen Beziehungen; Paraphile schätzen sich selbst als ängstlicher und konfliktvermeidender ein. Während ein Training sozialer Fertigkeiten wohl bei allen indiziert ist, müssten die Inhalte und Schwerpunkte des Trainings störungsbezogen variieren. Während die meisten Paraphilen Defizite in der Selbstbehauptung aufweisen, haben Impulskontrollgestörte eher Probleme mit Ärgerkontrolle und aggressivem Verhalten. Ein Problem bei der Behandlung kann sein, dass das unsichere und überangepasste Verhalten von Paraphilen nicht richtig «gedeutet» wird, dass ihr beobachtbares Verhalten als im Vergleich mit Impulskontrollgestörten angemessener angesehen wird usw. Richtig wäre hier zu erkennen, dass dieses Verhalten einen wesentlichen Hintergrund für die Delikte darstellt, weil es den Aufbau wirklich befriedigender Beziehungen und 
Tab. 4. Störungsspezifische Behandlungselemente: paraphile vs. impulskontrollgestörte Sexualdelinquenten

\begin{tabular}{lll}
\hline Behandlungselemente & Paraphile & Impulskontrollgestörte \\
\hline $\begin{array}{l}\text { Aufbau von Beziehung und Motivation } \\
\begin{array}{l}\text { Selbstverantwortung/ Opferempathie } \\
\text { (kognitive Umstrukturierung) }\end{array}\end{array}$ & $\begin{array}{c}\text { in manchen Fällen hoher Leidensdruck } \\
\text { kein generelles Empathiedefizit, } \\
\text { typische kognitive Verzerrungen } \\
\text { Stimuluskontrolle/Kontrolle } \\
\text { symptomatischen Verhaltens }\end{array}$ & schwer \\
$\begin{array}{l}\text { Training sozialer Fertigkeiten } \\
\text { Alternative Konfliktbewältigung }\end{array}$ & Schwerpunkt: Assertivität & häufig wenig spezifische Stimulussituationen \\
Rückfallprävention & statt «Flucht in Rückzug und Phantasie» & Schwerpunkt: Ärger, Aggression \\
Medikamente & indiziert, extramurale Erprobung nötig & statt «Flucht in Gewalt» \\
\hline
\end{tabular}

damit auch (indirekt) die Entwicklung einvernehmlicher sexueller Beziehungen erschwert.

Während bei Paraphilen der Schwerpunkt auf die Vermehrung von Handlungsalternativen zur Selbstbehauptung gelegt werden müsste, ist bei Impulskontrollgestörten eher alternative Konfliktbewältigung indiziert. Alternatives Konfliktverhalten kann anhand von Ereignissen im Klinikalltag vorbereitet und geübt werden. Mittels Verhaltensexperimenten können die dem abweichenden Verhalten zugrundeliegenden Annahmen (z.B. «Diskutieren bringt nichts») modifiziert werden.

Während alternative Konfliktbewältigung bei Paraphilen bedeuten würde, Ärger und Kränkung nicht nur zu kontrollieren (mit sich selbst abzumachen), liegen die Bewältigungsalternativen bei Impulskontrollgestörten z.B. im Stressbewältigungs- oder Entspannungstraining. Man könnte die Unterschiede der beiden diagnostischen Gruppen pointiert wie folgt kennzeichnen: Überfordernde soziale Konflikte führen bei Impulstätern häufiger zur «Flucht in Gewalt», bei Paraphilen - vermittelt über sozialen Rückzug - zur «Flucht in die deviante Phantasie». Ausagieren und zunächst nur phantasierte Wunschbefriedigung scheinen hier sich gegenüberstehende Bewältigungsmechanismen zu sein.

\section{Kognitive Umstrukturierung und Modifikation kognitiver Verzerrungen}

Besonders die Entwicklung von Opferempathie sollte in allen Therapien mit Sexualdelinquenten eine Rolle spielen, wobei insbesondere bei Impulskontrollgestörten speziell zu diesem Zweck entwickelte Gruppenprogramme erfolgreich sein dürften [Pithers, 1994]. Untersuchungen zeigen, dass die Behandlung von Paraphilen allerdings weitaus spezifischer sein muss. Sie weisen durchaus keinen generalisierten Mangel an Empathie auf [vgl. Hayashino et al., 1995]; diese ist isoliert auf das Delikt bezogen eingeschränkt und/oder unterliegt rückwirkend Tendenzen der Verleugnung und Minimalisierung. Auch können verzerrte Kognitionen vorkommen, z.B. im Sinne nachträglicher Rechtfertigung, Fehleinschätzung von Konsequenzen, Abwertung und Schuldattribuierung auf das Opfer. Nach unserer Einschätzung sind diese Minimalisierungstendenzen ubiquitär bei Delinquenten und differieren nur in der konkreten inhaltlichen Ausgestaltung. Es gibt keinen Beleg dafür, dass sie nicht mit gängigen kognitiven Methoden behandelt werden könnten.

\section{Rückfallprävention}

Ziel der Rückfallprävention ist das rechtzeitige Erkennen und Unterbrechen eines beginnenden offense cycle. Rückfallprävention ist die wohl wichtigste Methode in der Behandlung von Paraphilien. Paraphiles Verhalten weist Ähnlichkeiten zu süchtigem Verhalten auf; paraphile Phantasien und entsprechendes Verlangen können nie sicher ausgeschlossen werden. Strategien, den abstinence violation effect $\mathrm{zu}$ vermeiden bzw. trotz devianter Phantasien und Wünsche kein deviantes Verhalten zu zeigen, sind deshalb in der Behandlung wesentlich. Relevante Teilstrategien sind die Förderung der Selbstregulation und Methoden der Stimuluskontrolle. Vermeidung von Kontakt zu Kindern, von einschlägigem Pornographie-Konsum, von Internetkontakten usw. sind Beispiele für Stimuluskontrolle bei Paraphilen. Der Stand der Forschung lässt auch zu diesem Therapieelement noch keine valide Einschätzung zu, inwieweit es sich um ein effektives therapeutisches Instrument handelt. Es wurde aber im Rahmen integrativer Behandlungsmodelle bereits erfolgreich eingesetzt [vgl. Frenken, 1994].

Rückfallprävention in der Behandlung von Impulskontrollgestörten ist weniger spezifisch; es geht hier eher um die Identifikation und Modifikation von Risikofaktoren für eine Bandbreite gewalttätiger oder krimineller Verhaltensweisen. Stimuli, die das Gemisch von aggressiv-sexuellem Verhalten der Impulskontrollgestörten auslösen, sind schwer einzugrenzen; der Stationsalltag kann allerdings auch hier Hinweise für den 
Einsatz und das Erlernen von Stimuluskontrolle bieten, z.B. im Zusammenhang mit Alkoholkonsum.

Unterschiede in den Störungsmerkmalen von Paraphilen und Impulskontrollgestörten und die daraus resultierenden Behandlungsvorschläge haben wir in der Tabelle 4 verkürzt zusammenfasst.

\section{Abschließende Bemerkungen}

Man kann die hier vorgestellten Überlegungen auch als ein Forschungsprogramm auffassen. Weniges von dem, was wir hier auf der Basis der vorliegenden Literatur deduziert haben, auch nicht unsere Vorschläge zur differentiellen Behandlung, ist ausreichend empirisch abgesichert. Wir halten es aber für besser, bereits in einem frühen Forschungsstadium Vorschläge zur störungsspezifischen Behandlung zu machen, allein schon um dem für Forschung, Therapie und Prognose unangemessenen Denken entgegenzuwirken, «gefährliche» Sexualdelinquenten seien eine homogene Gruppe.

Im Sinne einer vereinfachenden Darstellung haben wir hier einige Fragezeichen vernachlässigt, die hinter einer verhaltenstherapeutischen Behandlung im Maßregelvollzug normalerweise gemacht werden. Die Therapiemotivation - besser: die Veränderungsmotivation - ist dort häufig gering bzw. mit Fremdmotivation vermischt [vgl. Gütlich et al., 1998]; das therapeutische Setting ist keineswegs das Günstigste, sondern stellt einen Kompromiss zwischen reiner Sicherung und therapeutischen Möglichkeiten dar. Schließlich wäre die Frage der Komorbidität zu berücksichtigen. Besonders bei schwerwiegenden Persönlichkeitsstörungen, gerade antisozialer Prägung, ist die Chance auf Therapieerfolg auch bei sonst aussichtsreichen Therapieelementen herabgesetzt [vgl. Lösel und Bender, 1997].

Eine weitere Komplikation ist, dass es eine Kombination von Paraphilie und Impulsivität geben kann. Manche Paraphilien werden von den Betroffenen als sie plötzlich überwältigende Impulse erlebt. Diese Fälle dürfen bei der Differentialdiagnose nicht übersehen werden.

Trotz dieser Einschränkungen ist aber davon auszugehen, dass die psychotherapeutische Behandlung sexueller Gewalttäter wirkt, wenn auch eher im Bereich «kleiner» statistischer Effekte [vgl. Hall, 1995; Schorsch et al., 1985]. Unser Plädoyer geht dahin, dass man die Wirksamkeitsrate erhöht, wenn man die Möglichkeiten einer fundierten psychologisch-psychiatrischen Diagnostik stärker nutzt und auf dieser Grundlage die «passenden», die störungsspezifischen Behandlungselemente heranzieht.

\section{Literatur}

Abel GG, Mittelman M, Becker JV, Rathner J, Rouleau JL: Predicting child molesters response to treatment. Ann NY Acad Sci 1988;528:223-234.

Abel GG, Osborn C: The paraphilias: The extent and nature of sexually deviant and criminal behavior. Psychiatr Clin North Am 1992;15:675-687.

Abel GG, Osborn CA, Anthony D, Gardos P: Current treatments of paraphiliacs. Annual Rev Sex Res 1992; 3:255-290.

Amann G, Wipplinger R (Hrsg): Sexueller Missbrauch. Tübingen, dgvt, 1997.

Barbaree HE: Stimulus control of sexual arousal: Its role in sexual assault; in Marshall WL, Laws DR, Barbaree HE (eds): Handbook of Sexual Assault. New York, Plenum Press, 1990, pp 115-142.

Barbaree HE, Marshall WL: Erectile responses amongst heterosexual child molesters, father-daughter-incest-offenders and matched non-offenders: Five distinct age preference profiles. Can J Beh Sci 1989;21: 70-82.

Barbaree HE, Marshall WL, Lanthier RD: Deviant sexual arousal in rapists. Behav Res Ther 1979;17:215222.

Barratt ES: The Barratt Impulsiveness Scale (BIS11). The University of Texas Medical Branch, 1995. Baxter DJ, Barbaree HE, Marshall WL: Sexual responses to consenting and forced sex in a large sample of rapists and non-rapists. Behav Res Ther 1986;24: 513-520.

Berner W, Berger P, Guitierrez K, Jordan B, Berger K: The role of personality disorders in the treatment of sex offenders. J Offender Rehabil 1992;18:25-37.
Berner W, Karlick-Bolten E: Vergleich zwischen «Paraphilie» und «sexuellen Impulshandlungen» bei Sexualdelinquenten. Forensia 1985;5:157-173.

Brown C: Paraphilias. Sadomasochism, fetishism, transvestism and transsexuality. Br J Psychiatry 1983; 143:227-231.

Fiedler P: Therapieplanung in der modernen Verhaltenstherapie. Verhaltensther Verhaltensmed 1997;18 7-39.

Fiedler P: Persönlichkeitsstörungen: Schlüssel zum Verständnis therapeutischer Krisen; in Zielke $\mathrm{M}$ (Hrsg): Handbuch stationäre Verhaltenstherapie, Weinheim, Psychologie-Verlags-Union,1994, pp 785 795.

Frenken J: Treatment of incest perpetrators: A fivephase model. Child Abuse Negl 1994;18:357-365.

Füllgrabe U: «Sexual»verbrechen aus Gewaltmotivation. BASIC ID - Die realitätsgerechte Analyse menschlichen Verhaltens. Kriminalistik 1994;48: 241247.

Fydrich T, Schmitz B, Hennch C Bodem M: Zuverlässigkeit und Gültigkeit diagnostischer Verfahren zur Erfassung von Persönlichkeitsstörungen; in Schmitz B, Limbacher K (Hrsg): Persönlichkeitsstörungen: Diagnostik und Psychotherapie. Weinheim, PsychologieVerlags-Union, 1996, S91-116.

Gütlich V, Heidenreich T, Hoyer J: Stadien der Veränderung und Therapiemotivation unter justiziellem Zwang. Verhaltenstherapie Psychosoz Prax 1998;30: 421-432.
Hall GCN: Sex offender recidivism revisited: A metaanalysis of recent treatment studies. J Consult Clin Psychol 1995;63:802-809.

Hayashino DS, Wurtele SK, Klebe KJ: Child molesters - an examination of cognitive factors. J Interpers Violence 1995;10:106-116.

Horn HJ: Antiandrogene bei Sexualdelinquenz; in Wille R, Schumacher W, Andrzejak N (Hrsg): Zur Therapie von sexuell Devianten. Berlin, Diesbach, 1990.

Horowitz LM, Strauß B, Kordy H: Inventar zur Erfassung interpersonaler Probleme (IIP). Weinheim, Beltz, 1994

Hoyer J: Dysfunktionale Selbstaufmerksamkeit: Konstrukt und erste Ergebnisse zu seiner Erfassung. Frankfurt, Johann Wolfgang Goethe-Universität, 1996

Hoyer J, Kunst H, Borchard B, Stangier U: Paraphile versus impulskontrollgestörte Sexualdelinquenten: Eine psychologisch valide Differenzierung? Z Klin Psychol 1999;28:37-44.

Hoyndorf S, Christmann F: Verhaltenstherapie mit Sexualtätern; in Zielke M (Hrsg): Handbuch stationäre Verhaltenstherapie, Weinheim, Psychologie-VerlagsUnion, 1994, pp 774-784.

Hoyndorf S, Reinhold M, Christmann F: Behandlung sexueller Störungen. Ätiologie, Diagnostik, Therapie: Sexuelle Dysfunktionen, Mißbrauch, Delinquenz. Weinheim, Beltz/PVU, 1995. 
Jacobs S, Hoyer J, Katthän U, Zahnow U: Psychotherapie mit psychisch kranken Delinquenten. GWGZeitschrift 1990;80:223-227.

Kockott G: Sexuelle Störungen; in Margraf J (Hrsg) Lehrbuch der Verhaltenstherapie. Berlin, Springer, 1996, pp 295-318.

Kröber HL: Strafrechtliche Begutachtung von Persönlichkeitsstörungen. Persönlichkeitsstörungen 1997;1: 161-170.

Laws DR, Marshall WL: A conditioning theory of the etiology and maintenance of deviant sexual preference and behavior; in Marshall WL, Laws DR, Barbaree HE (eds): Handbook of Sexual Assault: Issues, Theories and Treatment of the Offender. New York, Plenum Press, 1990, pp 209-230.

Laws DR, Marshall WL: Masturbatory reconditioning with sexual deviants: An evaluative review. Adv Behav Res Ther 1991;13:13-25.

Lösel F, Bender D: Straftäterbehandlung: Konzepte, Ergebnisse, Probleme; in Steller M, Volbert R (Hrsg) Psychologie im Strafverfahren. Bern, Huber, 1997, S 171-204.

Marshall WL, Barbaree HE: An integrated theory o the etiology of sexual offending; in Marshall WL Laws DR, Barbaree HE (eds): Handbook of Sexual Assault: Issues, Theories and Treatment of the Offender. New York, Plenum Press, 1990, pp 257-278.
Marshall WL, Laws DR, Barbaree HE: Handbook of sexual assault: Issues, Theories and Treatment of the Offender. New York, Plenum Press, 1990.

Okami P, Goldberg A: Personality correlates of pedophilia: Are they reliable indicators? J Sex Res 1992 29:297-328.

Pithers WD: Process evaluation of a group therapy component designed to enhance sex offenders empathy for sexual abuse survivors. Behav Res Ther 1994; 32:565-570.

Quinsey VL, Chaplin TC, Upfold D: Sexual arousal to nonsexual violence and sadomasochistic themes among rapists and non-sex-offenders. J Consult Clin Psychol 1984;52:651-657.

Raine A: The Psychopathology of Crime. San Diego, Academic Press, 1993.

Rehder U: Klassifizierung inhaftierter Sexualdelinquenten - 1.Teil: Wegen Vergewaltigung und sexueller Nötigung Erwachsener Verurteilte. Mschr Krim Strafrechtsreform 1996a;79:291-304.

Rehder U: Klassifizierung inhaftierter Sexualdelinquenten - 2.Teil: Wegen sexuellen Mißbrauchs von Kindern Verurteilte. Mschr Krim Strafrechtsreform 1996b;79:373-385.

Saß H, Wittchen HU, Zaudig M: Diagnostisches und statistisches Manual psychischer Störungen (DSM IV). Göttingen, Hogrefe, 1996.
Schorsch E, Becker N: Angst, Lust, Zerstörung. Hamburg, Rowohlt, 1977.

Schorsch E, Galedary G, Haag A, Hauch M, Lohse H: Perversion als Straftat. Berlin, Springer, 1985

Schorsch E, Pfäfflin F: Die sexuellen Deviationen und sexuell motivierte Straftaten; in Venzlaff U, Foerster K (Hrsg): Psychiatrische Begutachtung. Stuttgart, Gustav Fischer, 1994, pp 323-368.

Schüler-Springorum H, Berner W, Cirullies B, Leygraf N, Nowara S, Pfäfflin F, Schott M, Volbert R: Sexualstraftäter im Maßregelvollzug - Grundfragen ihrer therapeutischen Behandlung und der Sicherheit der Allgemeinheit. Mschr Krim Strafrechtsreform 1996; 79:147-201.

Schumacher W: Zur Typologie und Dynamik delinquenter Sexualabweichungen; in Wille R, Schumache W, Andrzejak N (Hrsg): Zur Therapie von sexuell Devianten. Berlin, Diesbach, 1990, pp 1-8.

Seligman ME: What you can change...and what you can't. New York, Fawcett, 1993.

Stangier U, Heidenreich T: Diagnostik der Sozialen Phobie. Verhaltenstherapie 1997;7:107-118.

Ward T, Hudson SM, Marshall WL: Cognitive distortions and affective deficits in sex offenders: A cognitive deconstructionist interpretation. Sexual Abuse. J Res Treatment 1995;7:67-83.

Wulfert E, Greenway DE, Dougher MJ: A logical functional analysis of reinforcement based disorders: Alcoholism and paedophilia. J Consult Clin Psychol 1996;64:1140-1151 